\title{
Eine seltene Ursache pulmonaler Rundherde
}

\author{
Michael Tsuyoshi Chew ${ }^{\mathrm{a}} \quad$ Eric Chak $^{\mathrm{b}}$ Karen Matsukuma $^{\mathrm{c}}$ \\ a Department of Internal Medicine, University of California at Davis, Sacramento, CA, USA; \\ ${ }^{b}$ Department of Gastroenterology, University of California at Davis, Sacramento, CA, USA; \\ 'Department of Pathology and Laboratory Medicine, University of California at Davis, Sacramento, CA, USA
}

\author{
Schlüsselwörter \\ Pulmonale Rundherde · Morbus Crohn . \\ Extraintestinale Manifestationen
}

\section{Zusammenfassung}

Morbus Crohn ist eine chronische idiopathische Autoimmunerkrankung, die hauptsächlich den Gastrointestinaltrakt (GIT) befällt. Charakteristisch für die Erkrankung ist eine transmurale Entzündung, die überall im Verdauungstakt vom Mund bis zum After auftreten kann. Darüber hinaus sind bei M. Crohn nicht selten extraintestinale Manifestationen (EIM) zu beobachten, die nahezu alle Organsysteme betreffen können. Schätzungen zufolge kommt es bei bis zu 36\% der M. Crohn-Patienten zu EIM, allerdings schwanken die Angaben zur Inzidenz und Prävalenz der Lungenbeteiligung in der Literatur stark, mit Werten von teilweise nur 0,4\%. Es gibt nur wenige Fallberichte über pulmonale Manifestationen, da diese oftmals übersehen werden, insbesondere dann, wenn die respiratorischen Symptome vor Diagnose der gastrointestinalen Manifestationen aufgetreten sind, so wie im vorliegenden Fall. Eine 44-jährige ansonsten gesunde Frau stellte sich mit unspezifischen respiratorischen Beschwerden und rezidivierenden Pneumonien vor. In den mitgebrachten Computertomographie-Aufnahmen war eine diffuse, migratorische noduläre und konsolidierende parenchymale Lungenerkrankung zu erkennen; die infektiologische und rheumatologische Untersuchung war weitgehend unauffällig. Die Lungenbiopsie ergab nekrotisierende und nicht-nekrotisierende Granulome, sodass der Verdacht auf eine Sarkoidose aufkam. In den anschließenden bildgebenden Untersuchungen fand sich als Zufallsbefund eine Geschwulst im Caecum. Die Biopsie der Caecum-Geschwulst zeigte eine akute Kryptitis, einen Kryptenabszess und ein einzelnes schwach ausgebildetes Granulom, die auf M. Crohn hindeuteten. Im vorliegenden Bericht stellen wir eine Patientin vor, deren pulmonale Manifestationen schließlich zur Diagnose M. Crohn führten.

C 2016 S. Karger GmbH, Freiburg

\section{Einleitung}

Morbus Crohn ist eine entzündliche Systemerkrankung, die überwiegend den Gastrointestinaltrakt befällt. Darüber hinaus kommt es bei M. Crohn nicht selten zu extraintestinalen Manifestationen (EIM), die nahezu alle Organsysteme betreffen können. Diese EIM können unterschiedlich schwer ausfallen und den Patienten stärker beeinträchtigen als die Grunderkrankung. Manche EIM folgen in ihrem Verlauf der Aktivität des M. Crohn, während andere vollkommen unabhängig davon verlaufen. Da
EIM nahezu alle Organsysteme betreffen können, ist das klinische Bild sehr variabel. Sehr gut beschrieben sind unter anderem folgende EIM: Augenbeteiligung (Uveitis und Episkleritis), Hautbeteiligung (Erythema nodosum und Pyoderma gangraenosum), Manifestationen am Bewegungsapparat (reaktive Arthritis und Spondyloarthritis) sowie Leber- und Gallenwegserkrankungen [1]. Pulmonale Manifestationen sind weniger bekannt, können jedoch sowohl die Atemwege als auch das Parenchym betreffen.

\section{KARGER}

Fax +497614520714

information@karger.com

www.karger.com

\section{() 2018 S. Karger GmbH, Freiburg}

Accessible online at:

www.karger.com/kkp
Michael Tsuyoshi Chew

Department of Internal Medicine

University of California at Davis

4150 V Street, Suite 3100, Sacramento, CA 95817, USA

mtchew@ucdavis.edu 

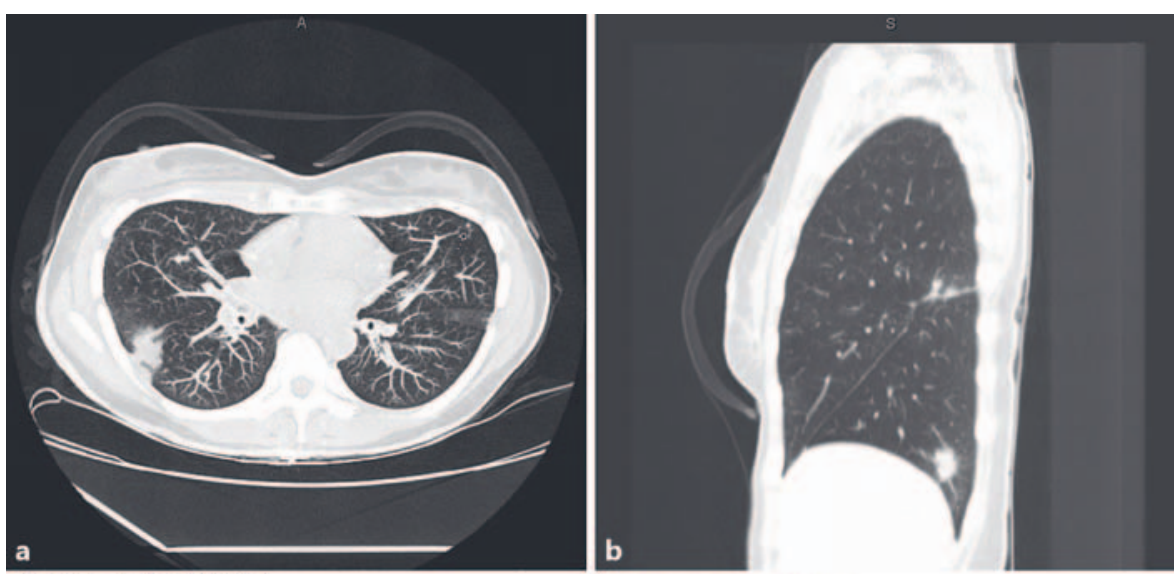

Abb. 1. Axiale (a) und sagittale (b) Computertomographie-Aufnahmen des Thorax mit multiplen pulmonalen Rundherden. (c) PET-Scan mit Fluordesoxyglucose (FDG)-aviden pulmonalen Rundherden im linken Unterlappen und assoziierten pleuralen Ausziehungen. (d) FDGAnreicherung in einer Raumforderung, die der Wand des Caecums entspringt.
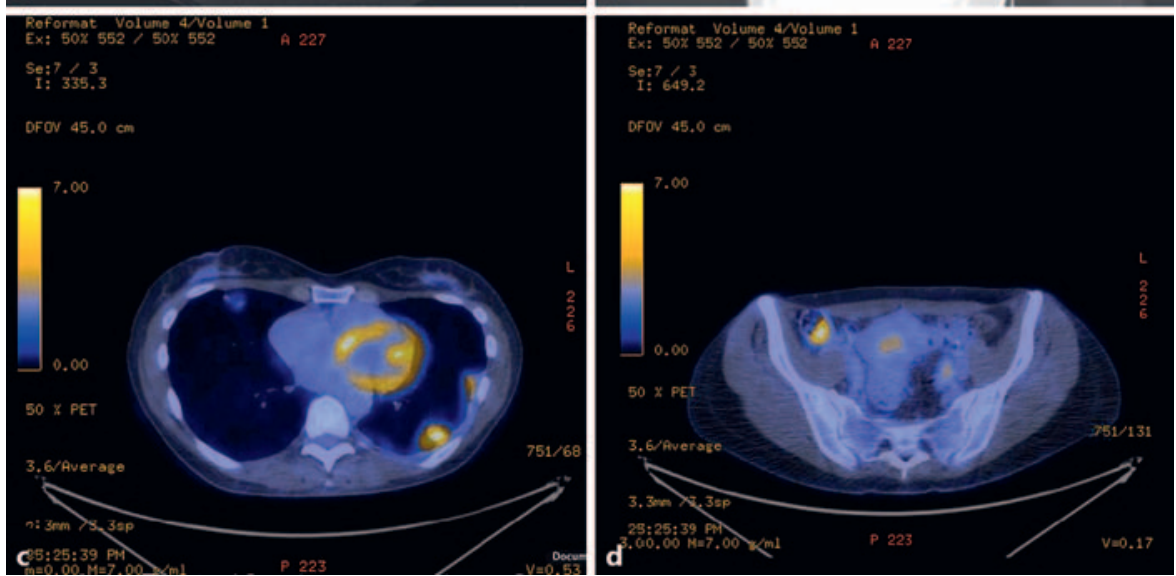

Da eine Lungenbeteiligung bei $\mathrm{M}$. Crohn selten ist, kann die Diagnose bisweilen schwierig sein. Zudem wird der Zusammenhang leicht übersehen, wenn die Lungenerkrankung unabhängig von der gastrointestinalen Erkrankung verläuft, und insbesondere dann, wenn die Beurteilung durch Spezialisten unterschiedlicher Fachrichtungen erfolgt. In der vorliegenden Kasuistik berichten wir zusammenfassend von einer Patientin mit Manifestationen, die wir im Nachhinein als pulmonale EIM vor Diagnose eines M. Crohn einstufen und die in der aktuellen Literatur nicht umfassend beschrieben ist.

\section{Fallbericht}

Eine 44-jährige Frau wurde initial 2012 wegen rezidivierender Pneumonien, anhaltender Kurzatmigkeit und Thoraxschmerzen in die Lungenklinik eingewiesen. Ihren Angaben zufolge hatte der klinische Verlauf 2007 begonnen, als bei ihr Asthma im Erwachsenenalter diagnostiziert worden war. Bis 2011 waren ihre Symptome relativ leicht. Dann wurde eine Pneumonie diagnostiziert und sie erhielt eine Behandlung mit Antibiotika und Kurzzeittherapien mit Prednison. Trotz Behandlung hatte sie das Gefühl, sich nie wieder vollständig erholt zu haben. Es kam weiterhin episodisch zu Belastungsdyspnoe und pleuritischen Thoraxschmerzen, die während der nächsten eineinhalb Jahre von rezidivierenden Pneumonien verkompliziert wurden.

Die körperliche Untersuchung der jungen und gesund erscheinenden Patientin war unauffällig. Skleren und Konjunktiven waren normal, die Schleimhaut im Nasenrachenraum erschien jedoch leicht gerötet. Die Herzuntersuchung war unauffällig und beide Lungenflügel waren auskultatorisch frei. Die Bauchdecke war weich und nicht druckschmerzhaft und es fanden sich keine Zeichen einer Haut- oder Gelenkbeteiligung.

Bei der Erstuntersuchung zeigte sich eine normozytäre Anämie (Hämoglobin 11,4 g/dl) mit peripherer Eosinophilie (1300/ $\mathrm{mm}^{3}$ ). In der Lungenfunktionsuntersuchung war bei der Patientin ein gemischt obstruktiv-restriktives Muster mit reduzierter Diffusionskapazität der Lunge für Kohlenmonoxid $\left(\mathrm{D}_{\mathrm{LCO}}\right)$ zu beobachten, und in mehreren CT-Untersuchungen zeigte sich eine diffuse, migratorische, noduläre und konsolidierende parenchymale Lungenerkrankung (Abb. 1a, b). Die erhöhten Entzündungsmarker standen im Einklang mit den deutlichen Symptomen einer Belastungsdyspnoe und den pleuritischen Thoraxschmerzen (Erythrozytensedimentationsrate: $32 \mathrm{~mm} / \mathrm{h}$; C-reaktives Protein: 2,3 mg/dl). Die infektiologische Untersuchung war positiv für Aspergillus-Präzipitine, jedoch negativ für Coccidioidomykose, Histoplasmose und Strongyloides ebenso wie der QuantiFERONAssay und das HIV-Screening. Die Bronchoskopie ergab keine Hinweise auf eine Infektion oder Eosinophilie. Eine eingehende serologische Untersuchung ergab schwach positive antinukleäre Antikörper mit einem Titer von 1:80 und IgE-Werten zwischen 200 und 300, war ansonsten jedoch negativ. Um die pulmonalen Läsionen besser charakterisieren zu können, wurde ein PET-Scan durchgeführt, der bilaterale pleurale und parenchymale Knoten zeigte, von denen der größte im linken Unterlappen lokalisiert 
Abb. 2. (a) Lungenbiopsie mit 3 Granulomen (Pfeile). (b)

Lungenbiopsie mit zentraler Nekrose (*), Makrophagen (**) und kleinen nichtnekrotisierenden Granulomen $(* * *)$. (c) Lungenbiopsien mit eitriger (neutrophiler) zentraler Nekrose. (d) Caecum-Biopsie unter geringer VergröBerung (der Pfeil zeigt auf ein angedeutet ausgebildetes Granulom). (e) Caecum-Schnitte mit akuter Kryptitis und Kryptenabszess. (f)

Caecum-Schnitte unter starker Vergrößerung mit angedeutet ausgebildetem Granulom. Pfeil, Makrophagen. *Eosinophile.
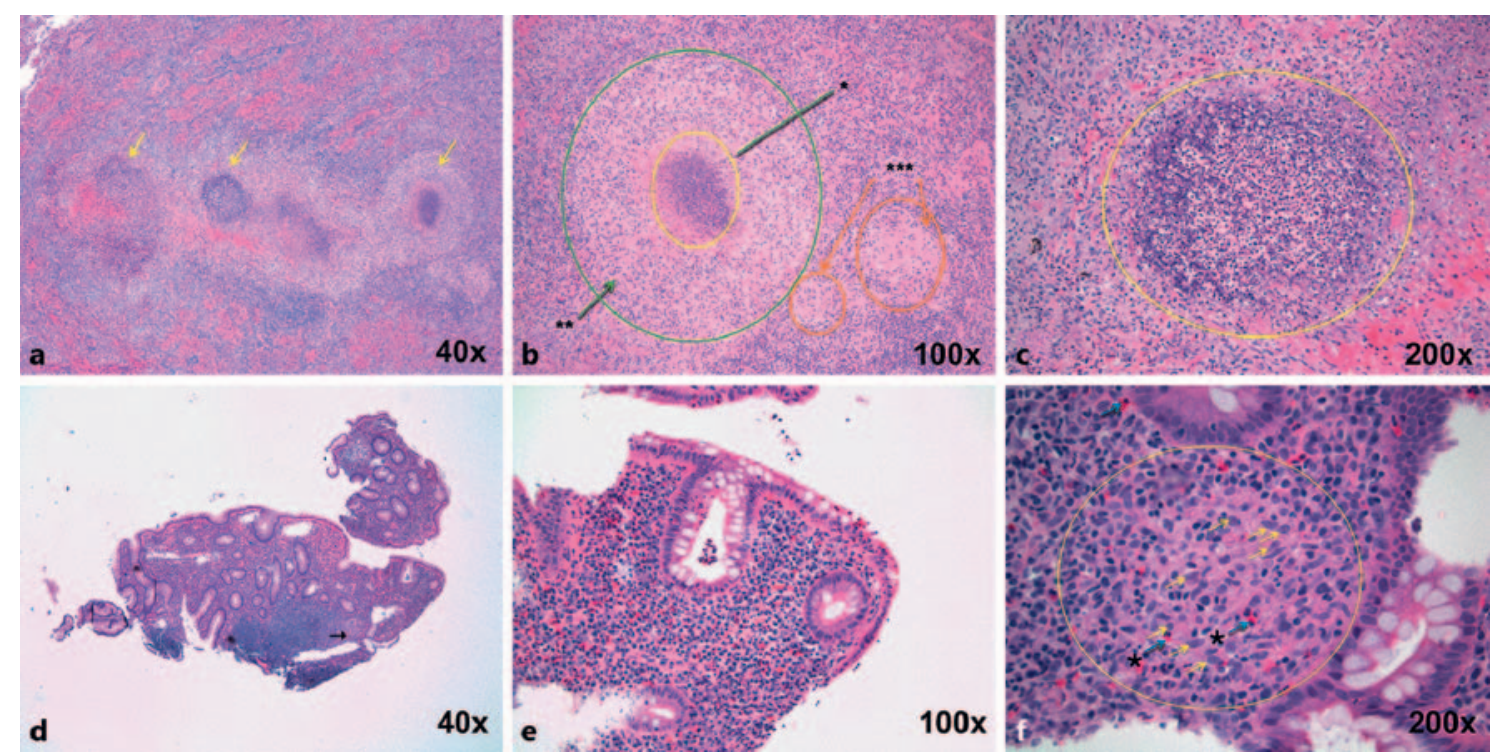

war (Abb. 1c). Als Zufallsbefund fand sich eine Geschwulst in der Caecumwand (Abb. 1d). Die CT-gesteuerte Biopsie des linken Unterlappens ergab eine prädominante Nekrose knapp umgeben von Granulationsgewebe, die negativ für säurefeste Bazillen und frei von Mikroorganismen war. Aufgrund der unspezifischen Ergebnisse erfolgte 2013 schließlich eine videoassistierte thorakoskopische chirurgische Lungenbiopsie des rechten Ober-, Mittelund Unterlappens, die eine Kombination von nekrotisierenden und nicht-nekrotisierenden Granulomen, eine neutrophile Entzündung und verstreute Eosinophile zeigte (Abb. 2a-c). Die Biopsie-Ergebnisse legten den Verdacht auf eine chronische idiopathische granulomatöse Lungenerkrankung nahe und es wurde entschieden, eine Behandlung mit Prednison in mittlerer Dosierung zu beginnen und über sechs Monate auszuschleichen. Trotz Abklingen der Symptome gelang es nicht, Prednison auszuschleichen. Mit dem Absetzen der Therapie traten die Symptome erneut auf; die Patientin klagte über anhaltende pleuritische Thoraxschmerzen, Belastungsdyspnoe, Kopfschmerzen, Fatigue und diffuse Arthralgien.

Die behandelnden Ärzte waren über die trotz Behandlung bestehende Symptomatik irritiert. Bei der eingehenden Überprüfung der Voruntersuchungen fiel das Augenmerk auf den PETScan-Nachweis einer Geschwulst der Caecumwand, dem zuvor nicht nachgegangen worden war, da die Patientin keine signifikanten gastrointestinalen Symptome hatte. Im September 2014 erfolgte eine Koloskopie, die eine isolierte nodulär veränderte und brüchige Caecumwand sowie ein fleckiges Erythem im Rektum zeigte. In der Biopsie der Caecum-Geschwulst fanden sich eine akute Kryptitis, ein Kryptenabszess und ein einzelnes schwach ausgebildetes Granulom, die differentialdiagnostisch M. Crohn vermuten ließen (Abb. 2d-f). Es wurde eine Behandlung mit Infliximab und 6-Mercaptopurin begonnen, unter der es zu einer signifikanten Besserung kam.

\section{Diskussion}

M. Crohn ist eine chronische idiopathische entzündliche Autoimmunerkrankung. Charakteristisch für die Erkrankung ist eine transmurale Entzündung, die überall im Verdauungstakt vom Mund bis zum After auftreten kann. Schätzungen zufolge kommt es bei 21-36\% der M. Crohn-Patienten zu EIM, wobei die Angaben zur Inzidenz und Prävalenz der Lungenbeteiligung in der Literatur stark schwanken [1]. Pulmonale Manifestationen von M. Crohn wurden erstmals in den späten 1960er Jahren beschrieben, doch erst Mitte der 1970er Jahre wurden sie in der Literatur allgemein als EIM einer chronisch entzündlichen Darmerkrankung (CED) anerkannt [2-5]. Eine pulmonale Beteiligung kommt bei M. Crohn nicht häufig vor und wird oftmals übersehen, insbesondere dann, wenn die respiratorischen Symptome vor Diagnose der gastrointestinalen Manifestationen aufgetreten sind, so wie im vorliegenden Fall. Man nimmt an, dass keine Darmentzündung vorliegen muss, damit respiratorische Symptome auftreten, da embryologische Gemeinsamkeiten zwischen dem respiratorischen und dem Kolonepithel bestehen [6]. Die Lunge und der Gastrointestinaltrakt weisen ein ähnliches submuköses lymphoides Gewebe auf, das für die Wirtsabwehr, aber auch bei der Entstehung einer entzündlichen Fehlregulation eine entscheidende Rolle spielt $[3,6,7]$.

Die meisten pulmonalen EIM treten Jahre nach der Diagnose einer CED auf [8]. Im vorliegenden Fall wurde die Diagnose jedoch dadurch verzögert, dass die pulmonalen Symptome der CEDDiagnose vorausgingen. Soweit uns bekannt, handelt es sich um einen der seltenen Fälle, in denen bei einem erwachsenen Patienten zuerst pulmonale EIM auftraten und erst dann die Diagnose einer CED gestellt wurde $[6,8,9]$. Die serologischen Untersuchungen auf eine CED fielen negativ aus; diese wurde erst durch eine Caecum-Biopsie bestätigt. Sowohl bei M. Crohn als auch bei Sarkoidose kann es zur Entstehung nicht-verkäsender Granulome 
kommen. Wichtige differentialdiagnostische Befunde waren im vorliegenden Fall die für eine Sarkoidose untypische periphere Eosinophilie sowie die normalen ACE (Angiotensin-converting enzyme)-Serumlevel mit Normalwerten für Calcium im Serum und im 24 h-Sammelurin [10]. Die ACE-Serumkonzentrationen sind bei M. Crohn meist niedrig bis normal, insbesondere in Phasen aktiver Entzündung [9]. Die Patientin hatte eine normozytäre Anämie und ihre Eisenwerte sprachen für das Vorliegen einer Anämie bei chronischer Entzündung mit Eisenmangel (Ferritin: $8 \mathrm{ng} / \mathrm{ml}$; prozentuale Eisensättigung: 11\% und eine Erythrozytenverteilungsbreite von 17 Einheiten), wie sie bei M. Crohn zu beobachten ist, jedoch nicht typischerweise bei Sarkoidose [10]. Angesichts der negativen Ergebnisse der serologischen Untersuchungen, Kulturen und Spezialfärbungen der offenen Lungenbiopsie bestand nur ein geringer Verdacht auf infektiöse Ursachen. Die Lungenerkrankung passte nicht zu einer spezifischen nicht-infektiösen granulomatösen Lungenkrankheit. Wegen der granulomatösen Entzündung sowie der radiologischen Verteilung, der Asthma-Anamnese und der peripheren Eosinophilie wurde kurzzeitig eine Granulomatose mit Polyangiitis in Betracht gezogen. Die im vorliegenden Fall fehlende nekrotisierende Vaskulitis und die negative Anti-PR3-Serologie sprachen jedoch gegen die Diagnose Granulomatose mit Polyangiitis.

Wegen des heterogenen und breiten Spektrums der EIM ist die pulmonale EIM von M. Crohn noch immer unterdiagnostiziert. Im vorliegenden Fall gingen die pulmonalen Manifestationen dem Nachweis und letztlich der Diagnose M. Crohn voraus. Wie dieser Fall verdeutlicht sind umfassende infektiologische, rheumatologische und pathologische Untersuchungen sehr wichtig. Der Wendepunkt dieses Falls war gekommen, als wir entschieden, die murale Geschwulst im Caecum der Patientin endoskopisch und bioptisch näher zu untersuchen. Erst durch die diagnostische Abklärung ihres Colon-Befunds wurde festgestellt, dass es sich bei der Lungenerkrankung um eine Manifestation von M. Crohn handelt.

Zwar wurden in Studien Lungenfunktionsuntersuchungen bei CED-Patienten durchgeführt, doch fielen die Ergebnisse subklinisch und häufig gemischt aus. Ursache hierfür ist den Annahmen zufolge die nicht ausreichende Power der Studien. Im
Vergleich zu gesunden Probanden wiesen die CED-Patienten jedoch durchweg eine verminderte $\mathrm{D}_{\mathrm{LCO}}$ auf, und während der aktiven Phase könnte dieser Wert noch signifikant niedriger sein $[4,6]$. Kortikosteroide gelten als Hauptpfeiler der Behandlung einer pulmonalen EIM bei M. Crohn [11]. Allerdings sprechen bis zu einem Drittel der Patienten nicht auf die Therapie an oder ein Ausschleichen ist unmöglich, wie es auch bei dieser Patientin der Fall war. Es wurde eine Behandlung mit Infliximab und 6-Mercaptopurin begonnen, woraufhin sich die pulmonalen Symptome signifikant besserten. Nach einjähriger dualer Therapie wurde die Patientin auf eine Monotherapie mit Infliximab umgestellt, unter der es ihr weiterhin gut geht.

Die Quintessenz dieses Falls ist, dass sich Krankheiten nicht immer wie im Lehrbuch darstellen. Insbesondere können die bei $M$. Crohn auftretenden EIM sogar den erfahrensten Kliniker täuschen, vor allem, wenn die EIM allen deutlichen Entzündungszeichen und Symptomen einer gastrointestinalen Erkrankung vorausgeht. Nach gründlicher Abklärung ihrer pulmonalen Symptomatik veranlassten uns der PET-Scan der Patientin und ein Zufallsbefund, die Patientin eingehender auf CED zu untersuchen. Erst als die Diagnose M. Crohn feststand, stellten wir einen Zusammenhang her und erkannten, dass es sich bei der Lungenerkrankung um eine Manifestation der CED handelte.

Dank

Dieser ungewöhnliche Einzelfallbericht hätte ohne die Arbeit von Dr. Jason Adams vom Department of Pulmonary and Critical Care Medicine und Dr. Jesse Stondell vom Department of Gastroenterology, University of California at Davis, Sacramento, CA, USA, nicht identifiziert werden können.

\section{Erklärung zu ethischen Konflikten}

Vor der Veröffentlichung dieses Fallberichts hatte die Patientin schriftlich ihre Einwilligung erteilt.

\section{Disclosure Statement}

Die Autoren erklären, dass keine Interessenkonflikte bestehen.

\section{Literatur}

1 Juillerat P, et al.: Extraintestinal manifestations of Crohn's disease. Digestion 2007;76: 141-148.

2 Kraft SC, et al.: Unexplained bronchopulmonary disease with inflammatory bowel disease. Arch Intern Med 1976;136:454-459.

3 Lu DG, et al.: Pulmonary manifestations of Crohn's disease. World J Gastroenterol 2014; 20:133-141.

4 Songur N, et al.: Pulmonary function tests and high-resolution CT in the detection of pulmonary involvement in inflammatory bowel disease. J Clin Gastroenterol 2003;37:292-298.
5 Turner-Warwick M: Fibrosing alveolitis and chronic liver disease. Q J Med 1968;37:133-149.

6 Black H, Mendoza M, Murin S: Thoracic manifestations of inflammatory bowel disease. Chest 2007;131:524-532.

7 Bernstein CN, Wajda A, Blanchard JF: The clustering of other chronic inflammatory diseases in inflammatory bowel disease: a population-based study. Gastroenterology 2005;129: 827-836.

8 Casey MB, et al.: Noninfectious lung pathology in patients with Crohn's disease. Am J Surg Pathol 2003;27:213-219.
9 Levenbrown Y, et al.: Granulomatous lung disease as the initial presentation of Crohn disease. J Pediatr Gastroenterol Nutr 2009;48: 487-490.

10 Beniwal RS, Cummings OW, Cho WK: Symptomatic gastrointestinal sarcoidosis: case report and review of the literature. Dig Dis Sci 2003;48:174-178.

11 Hayek AJ, Pfanner TP, White HD: Inflammatory bowel disease of the lung: the role of infliximab? Respir Med Case Rep 2015;15: $85-88$. 